\title{
Iconic Memory, Location Information, and Partial Report
}

\author{
Siu L. Chow \\ University of Wollongong, Wollongong, New South Wales, Australia
}

It has been suggested that the systematic decline of partial report as the delay of the partial-report cue increases is due to a time-related loss of location information. Moreover, the backward masking effect is said to be precipitated by the disruption of location information before and after identification. Results from three experiments do not support these claims when new indices of location information and of item information are used. Instead, it was found that (a) the systematic decline in partial report was due to a time-related loss of item information, and (b) location information was affected neither by the delay of the partial-report cue nor by the delay of backward masking. Subjects adopted the

"select-then-identify" mode of processing.

Iconic memory, as a hypothetical mechanism, has an interesting and controversial history. Although its empirical foundation was established by Sperling (1960), it was so named by Neisser (1967) and integrated into the information-processing approach to perception by Haber (1969, 1971; Haber \& Hershenson, 1980). However, its importance is no longer recognized by Neisser (1976). Furthermore, its theoretical usefulness is now seriously questioned by Haber (1983) on metatheoretical grounds (in addition to other reasons). A metatheoretical rejoinder to Haber's (1983) metatheoretical argument has been offered by Loftus (1983) and Chow (in press).

At the theoretical level, iconic memory has been identified with (a) a kind of visible persistence that renders phenomenological report possible (Haber \& Nathanson, 1968; Haber \& Standing, 1969), (b) the aftereffects of stimulating the retinal rods (Sakitt, 1975, 1976a, 1976b), and (c) some sort of precategorical representation called "informational persistence" by Coltheart (1980). This article is concerned with the empirical foundation of iconic memory in the sense of informational persistence.

The empirical foundation of informational persistence is not without dispute. For example, Holding's (1970, 1972, 1975) critique of Sperling's (1960) partial-report paradigm in terms of some probable procedural artifacts, such as guessing and response selection, has been dealt with by Coltheart (1975, 1980). Merikle's (1980) questioning of the partial-report paradigm in terms of perceptual grouping has been answered by Chow (1985). It is necessary now to consider Mewhort and Butler's (1983) contention that the empirical basis of iconic memory is not sound in view of some findings by Mewhort and his associates (Campbell \& Mewhort, 1980; Mewhort \& Campbell, 1978; Mewhort, Campbell, Marchetti, \& Campbell, 1981). 
In Haber and Hershenson's (1980) theoretical account, information about the location, color, and size of stimuli is fully represented at the first stage of visual information processing. Moreover, when alphanumeric stimuli are involved, their representation at the retinal level is transformed into features, such as angles and straight lines; the persistence of these various types of information constitutes the iconic store. This view is consistent with Coltheart's notion of informational persistence. In the context of the partial-report task, a "select-then-identify" strategy is assumed. That is, only the bundle of features probed is selected for further processing to the item-identity level if the critical bundle is among those which have not been processed by the time the partial-report probe is presented. As the partial-report probe is delayed, the selected bundle becomes less useful because some or all of its features may have decayed.

In contrast, Mewhort et al. (1981) envisage an "identify-then-select" mode of operation in the partial-report situation. More specifically, they propose that the processing of visual stimuli requires the postulation of a feature buffer (preidentification) and a character buffer (postidentification). The selection of the probed item is carried out in the character buffer. To follow Mewhort et al.'s (1981) terminology, this model will subsequently be called the dual-buffer model.

Using Averbach and Coriell's (1961) version of the partial-report task, Mewhort et al. (1981) found that as the decay of the probe was increased, partial-report performance decreased together with a corresponding increase in location errors while item errors remained more or less constant. This observation suggested to Mewhort et al. (1981) that location information suffered when the partial-report cue was delayed. It further suggested that partial report was superior to whole report because "the [partial-report superiority] measure is heavily biased against full report. . ." (Mewhort \& Butler, 1983, p. 32).

When given Averbach and Coriell's (1961) task, the subject is presented with a multi-item array (e.g., $M B D H X L G$ ) which is subsequently followed by a partial-report probe after a predetermined delay. The subject is required to recall only the item indicated by the probe. That is, the criterion of selection is an intra-array location. With reference to the array, $M B D H Z L G, \mathrm{Z}$ is the correct response if Location 5 is probed. Under such circumstances, there are two kinds of errors. The subject may respond with an item in the array other than the correct one (e.g., $H$ ). This is an intra-array intrusion. Alternatively, the subject may respond with an item not present in the array (e.g., N). This is an extra-array intrusion. While acknowledging the possibility of some ambiguity Mewhort et al. (1981) nonetheless suggest that intra-array intrusions signify the loss of location information, whereas extra-array intrusions indicate the loss of item information.

Although it is straightforward to identify an intra-array intrusion, it is not easy to know what it signifies (a point also recognized by Mewhort et al., 1981, and Van der Heijden, 1984). For example, although Position 5 is probed, the subject may have mistaken it to be a probe for Position 4. That is, this is a misidentification of the probe's 
location, not a misidentification of the location of a particular item within the character buffer. If this is the case, it is inappropriate to consider responding with $H$ in the previous example as a location error at the level of the character buffer. In other words, it is necessary to test Mewhort et al.'s (1981) contention with another index of location information.

At the theoretical level, location information at the level of the character buffer plays an important part in the dual-buffer model. For example, location information must be represented in such a way that the relative location of the letters is preserved. Moreover, the representation must make it possible for ". . the attention mechanism [to find] an item in the character buffer by using the probe as a location instruction ..." (Mewhort et al., 1981, p. 51). Despite this important role played by location information, Mewhort et al. (1981) are vague about exactly how this location information is represented and used. Nonetheless, a particular type of representation of location information may be derived from their account.

It seems reasonable to suggest that the location information in the character buffer may assume the form of location tags for the items in the character buffer. That is, it is not inconsistent with the dual-buffer model to suggest that the location information of an identified item is tagged to the item in the character buffer. For example, $M$ at Location 1 of MBDHZLG may be represented as M-1 in the character buffer; $\mathrm{Z}$ as Z-2, and the like. When the partial-report probe is presented, the item having a location tag that matches the location of the partial-report probe is given as the response. That is, the essence of the identify-then-select mode of operation is that location information is accessed via item information, a mode of operation contrary to that envisaged in Treisman and Gelade's (1980) model.

As the partial-report probe is progressively delayed, fewer and fewer location tags remain useful, either as a result of decay or of their being transposed. For example, Z-5 might become Z-4, whereas $H-4$ may become $\mathrm{H}-5$. Hence, partial-report performance suffers, despite the fact that the information about the identities of the items has not changed. Consequently, responding with $H$ when the item occupying the fifth location of the array is probed may be categorized as a location error.

The conclusion drawn by Mewhort et al. (1981) is that item information decays from the character buffer at a much slower rate than location information. It follows from this conclusion that at any delay of the partial-report probe the probability that item information is available is higher than that of the availability of location information. When coupled with the identify-then-select assumption, several testable implications can be derived for Averbach and Coriell's (1961) partial-report task. However, the dependent variables to be used in this study have to be discussed first.

What measures can be used to reflect the availability of item and location information if Mewhort et al.'s (1981) measures are not satisfactory? This question has to 
be considered with reference to what the subject has to do. In the experiments to be reported, the subjects were given Averbach and Coriell's partial-report task and required to report both the letter probed as well as its intra-array location. First, consider the conditional probability of correct recall of item identity, given correct recall of its intra-array location (i.e., $p(\mathrm{I} \mid \mathrm{L})$, where I is the correct recall of item identity and $\mathrm{L}$ is the correct recall of its intra-array location). It has been noted that location information is accessed via item information if the identify-then-select mode of operation is in force. That is, $p(\mathrm{I} \mid \mathrm{L})$ represents the proportion of trials in which the correct recall of location is mediated by correct recall of item identity. Hence, the conditional probability, $p(\mathrm{I} \mid \mathrm{L})$, may be used as an index of the availability of item information if the dual-buffer model and the identify-then-select assumption are jointly adopted.

The second conditional probability of interest is the probability of correct recall of the intra-array location of the probe, given correct recall of item identity (i.e., $p[\mathrm{~L} \mid \mathrm{I}]$ ). This conditional probability is the proportion of trials in which the correct position information is still attached to the item. Otherwise, the recall of location will be incorrect because position information is always acessed via item information in the dual-buffer model. Hence, the conditional probability, $p(\mathrm{~L} \mid \mathrm{I})$, may be used as an index of the availability of location information. Some expectations in terms of $p(\mathrm{I} \mid \mathrm{L})$ and $p(\mathrm{~L} \mid \mathrm{i})$ may now be derived from the dual-buffer model.

Consider the no-masking condition. First, although its absolute level is uncertain, the conditional probability of item recall, given location recall (i.e., $p[\mathrm{I} \mid \mathrm{L}]$ should be constant, regardless of the delay of the partial-report probe (see the solid-line function in the top left panel of Figure 1). The second implication is that the conditional probability of location recall, given item recall (i.e., $p[\mathrm{~L} \mid \mathrm{I}]$, should decrease with increases in the interval between the offset of the stimulus and the onset of the probe, an interval called the interstimulus interval (ISI), regardless of its absolute level at ISI $=0 \mathrm{~ms}$ (see the solid-line function of the bottom left panel of Figure 1).

According to the dual-buffer model, the effect of backward masking at short mask delays is different from that at long mask delays. At short delays, the mask interrupts the features in the feature buffer, thereby bringing about problems in identification. That is, at short delays, masking increases item errors. If the mask is delayed sufficiently long, the system will have sufficient time to establish the characters in the character buffer. Hence, the mask interrupts only location information at the level of the character buffer. That is, masking increases location errors at long mask delays.

What should be expected from the dual-buffer model if a mask is presented concurrently with the probe in terms of the two conditional probabilities? It may first be suggested that in terms of $p(\mathrm{I} \mid \mathrm{L})$, the ISI function should increase with increases in ISI until an asymptote is reached; the function would remain at the asymptotic level with further increases in ISI (see the dotted line function in the top left panel of Figure 1). Second, in terms of $p(\mathrm{~L} \mid \mathrm{I})$, the ISI function should remain flat until a certain critical value 
of ISI is reached, after which the function would decrease steadily with increases in ISI (the dotted line function in the bottom left panel of Figure 1). That is, the two dotted line functions in the top-left and bottom-left panels of Figure 1 bend because the dual-buffer model envisages different backward masking effects at short and long mask-delays.
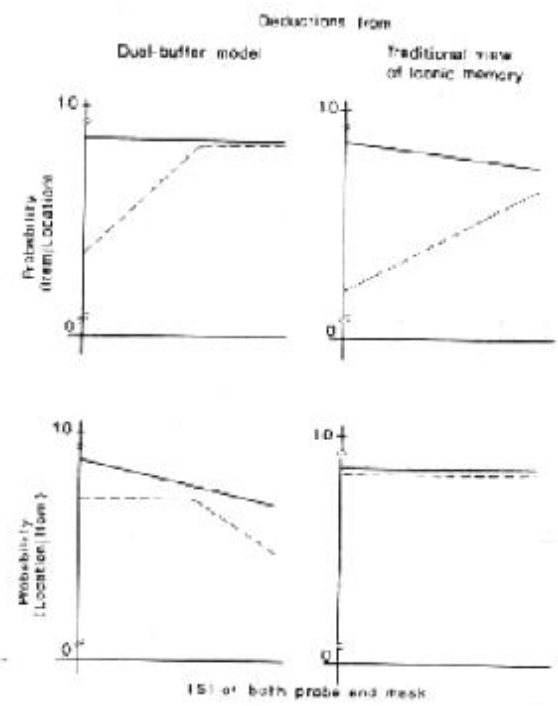

- The masting

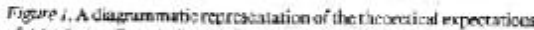

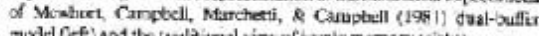

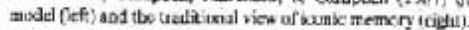

In the two panels on the right-hand side of Figure 1 are depicted the expectations of the orthodox view of iconic memory (Coltheart, 1977; Haber \& Hershenson, 1980). In the traditional view, the features extracted from the stimuli decay systematically with increases in ISI. The systematic decay results in a systematic decline in item information even though the infra-array location of the probed-for item is properly identified and recalled (see the solid line function in the top right panel of Figure 1). The reverse is expected when a mask is presented concurrently with the probe because masking interrupts further processing (Kahneman, 1968; Turvey, 1973). (See the dotted line function in the top right panel of Figure 1.)

The select-then-identify overtone of the traditional view implies that an item has to be properly located (selected) before it can be correctly recalled (see also Treisman \& Gelade, 1980), regardless of the ISI. Moreover, this situation is not affected by masking; hence, the pair of functions in the bottom right panel of Figure 1 is expected. Data from the first experiment to be reported will be assessed with reference to both Mewhort et al.'s (1981) model and the traditional view of iconic memory.

\section{General Method}


Because the three experiments to be reported followed the same general procedure, the method used is first presented in general terms. Specific departures from this general procedure will be described when applicable.

\section{Subjects}

A separate group of 14 subjects was run in each of the three experiments. Subjects in Experiments 1 and 2 were first-year psychology students who participated for two bonus marks. The group of subjects in Experiments 3 consisted of some undergraduate students and some members of the staff of the Department of Psychology at the University of Wollongong. All subjects were naive as to the theoretical expectations of the experiments. All subjects reported normal or corrected-to-normal vision.

\section{Apparatus}

Stimulus items were selected and presented with a PDP/I 1-23 microcomputer driving a CIT-100 CRT The type of phosphor used in the CRT is P-31 (green), which decays to the minimum discernible brightness in a brightly lit room in $38 \mu \mathrm{s}$, a condition used in this study. The stimulus duration and the ISI manipulation were timed with a programmable KW V I 1 A clock under the control of the program.

\section{Materials}

A seven-item array was presented to the subject on every trial. Seven consonants were selected randomly, without replacement, from an ensemble of 20 consonants. That is, the five vowels and the letter $\mathrm{Y}$ were not used in the experiment. A question mark was used as the partial-report probe. When applicable, a masking array (made up of seven grids of dots) was used as the mask.

Each letter was made up of 7 X 9 (points) grid subtending a visual angle of 20' horizontally and $38^{\prime}$ vertically. The seven-item array subtended a visual angle of $2^{\circ} 36^{\prime}$ horizontally and $38^{\prime}$ vertically

\section{Design}

A 2 X 5 complete factorial analysis of variance (ANOVA) with repeated measures on both factors was used in all three experiments. The first factor was the absence or presence of backward masking. The second factor was the ISI between the offset of the stimulus array and the onset of the partial-report probe and/or the mask. Because the three experiments differed in what was being delayed as well as the exact values for the five delay intervals, this factor will be described individually for each experiment.

\section{Procedure}


Subjects were advised that the purpose of the experiment was to establish how well they could perceive briefly presented visual stimuli in different conditions. They were tested individually in a I -hr session. The sequence of events in a trial was explained to the subjects with the help of a flow diagam. Each subject was trained with two 20-trial blocks of trials before the experiment. These practice blocks served to allow the subjects to get used to the brief stimulus duration and to be familiar with their experimental task.

A trial began with a warning tone. A fixation point (i.e., the $[+]$ symbol) was presented in the center of the CRT $1 / 2 \mathrm{~s}$ after the end of the warning tone. The duration of the fixation point was $500 \mathrm{~ms}$. A seven-item array appeared for a predetermined period $1 / 2 \mathrm{~s}$ after the offset of the fixation point. The fourth item of the stimulus array occupied the location previously occupied by the fixation point. The partial-report probe (i.e., the [?] symbol) was presented for $1 / 2 \mathrm{~s}$ at a predetermined ISI after the offset of the stimulus array. The probe was presented in the row above the seven-item array. Had the array and the probe been presented simultaneously, the probe would be above one of the seven letters in the array. When applicable, a masking array appeared at a predetermined delay after the offset of the stimulus array. The masking array stayed on until the subject had responded. The temporal relation between the partial-report probe and the masking array will be described individually for each experiment.

The subjects were required to recall the letter indicated by the probe as well as the letter's intra-array location. For example, if (?) appeared in the location above the fifth one of the array, MBDHZLG, the correct responses were $\mathrm{Z}$ and 5. The subjects typed in their responses. They were advised to take their time in order to be as accurate as possible. (The fact that subjects might not be equally proficient in typing was not a problem because reaction time was not measured. Moreover, it is reasonable to assume that typing proficiency would not interact with the two independent variables of interest.)

There were 240 trials in the experiment proper. Data from the first 30 trials were discarded because they were warm-up trials. The remaining 210 trials consisted of 21 trials of each of the 10 (2 masking levels X 5 ISI levels) treatment combinations. Among the 21 trials devoted to a treatment combination, each of the seven locations was probed three times. The 210 experimental trials were randomly selected, with replacement, from the 10 treatment combinations for individual subjects.

From the subjects' viewpoint, the session was divided into four blocks of 50 trials and one block of 40 trials (the last block). Within each block, the intertrial interval was 5 s. The duration of the break between blocks was controlled by the subjects.

A uniform level of Type I error, namely, 5\%, will be adopted throughout this report when the significance of statistical tests is reported.

\section{Experiment 1}


Mewhort et al. (1981) argued that location information is lost faster than item information in Averbach and Coriell's (1961) bar-probe task. Because there are reasons to question their choice of indices of loss of item information (i.e., extralist intrusion errors) and of loss of location information (i.e., intralist intrusion errors), this experiment was conducted to test Mewhort et al.'s (1981) dual-buffer model with two new indices.

The subjects were tested under both Conditions 1 and 2 of Mewhort et al.'s (1981) study in this experiment. That is, the partial-report probe and the mask (when applicable) were presented simultaneously. The ISI values used were $0,40,80,120$, and $160 \mathrm{~ms}$. The duration of the seven-item detection array was $20 \mathrm{~ms}$.

\section{Results}

In order to compare the results of this experiment with Mewhort et al.'s (1981) Conditions 1 and 2, the subjects' performance was scored in terms of intralist and extralist intrusion errors. As can be seen from the top left panel of Figure 2, masking increased the number of intralist intrusions, $F(l, 13) \_52.44$; so did increases in ISI, F $(4,52)=3.56$. Masking also increased the number of extralist intrusions, $F(1,13)=54.49$ (see top right panel of Figure 2). Although ISI did not have any effect on extralist intrusions, it interacted significantly with masking, $F(4,52)=3.86$.

As can be seen from the top panel of Figure 3, the subjects' performance in terms of the correct recall of both item identity and its location declines systematically as ISI increases under the no-masking condition. Masking reduced performance at short ISI levels. These observations were confirmed by ANOVA, which showed that the masking by ISI interaction was significant, $F(4,52)=5.49$. Also significant was the main effect of masking, $F(1,13)=110.71$. Tukey's HSD (honestly significant difference) test did not show any significant pairwise difference among the means of the five ISI levels under the masking condition. Represented in the middle and the bottom panels of Figure 3 are the conditional probabilities, $p(\mathrm{I} \mid \mathrm{L})$ and $p(\mathrm{~L} \mid \mathrm{I})$, respectively.

The middle panel of Figure 3 shows that more item information is available in the absence of masking than in its presence. As ISI increases, the availability of item information decreases under the no-masking condition. The ANOVA revealed that the masking by ISI interaction was significant, $\mathrm{F}(4,52)=6.16$; so was the main effect of masking, $F(1,13)=87.36$. Tukey's HSD test showed that the availability of item information did not vary as ISI increased under the masking condition, despite the apparently upward trend shown by the dotted-line function in the middle panel of Figure 3. A similar analysis for the availability of location information data did not reveal any signficant effect, as may be seen from the bottom panel of Figure 3. That is, the availability of location information did not vary with the delay of the probe, regardless of the masking manipulation. 
INTRALIST

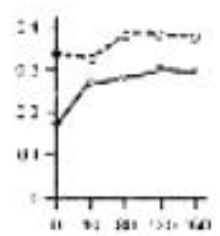

ISI QF PIOQE AND

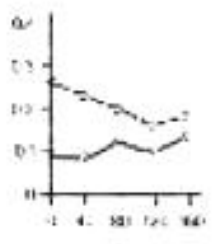

MASK (MSEC)

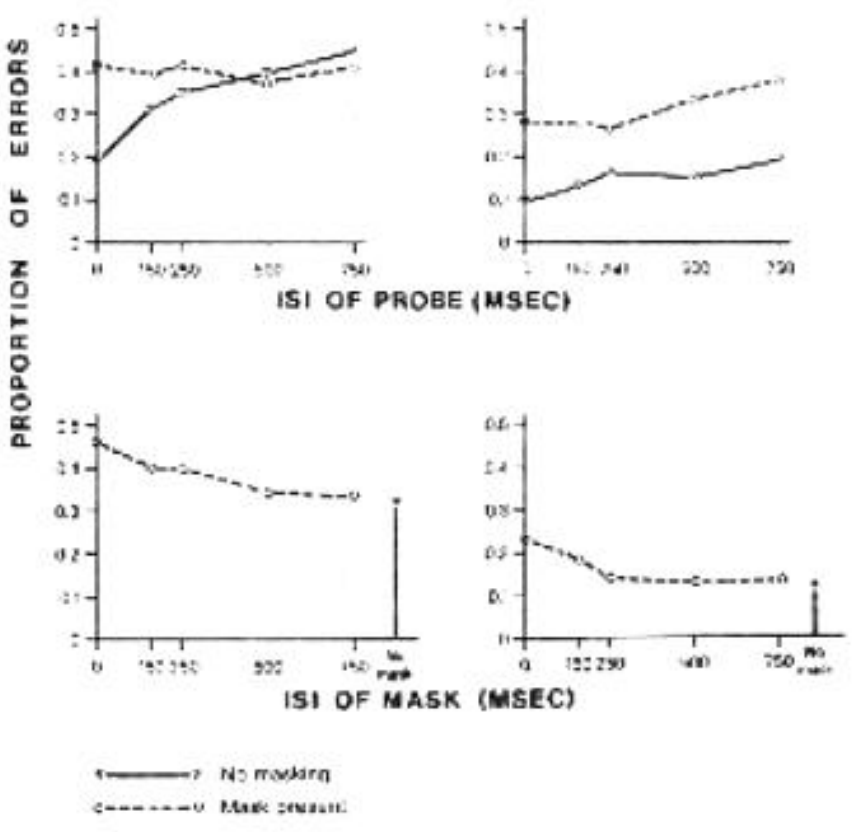

Figue 2 Number of intralist fleth atd extralist irighty antruswe error as a funetico of (s) the gynchmaized welay of probe and maxk (tip pauel:

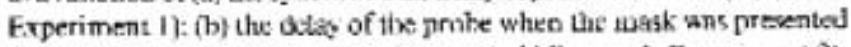
at tbe imostiate offet of the stimulus (middle janel; Expexitaent 2): and (c) the dowe of be mask when the probe was presented at the imubvdiste oficet of the stimulus (bottom panel: Experimeat 3)
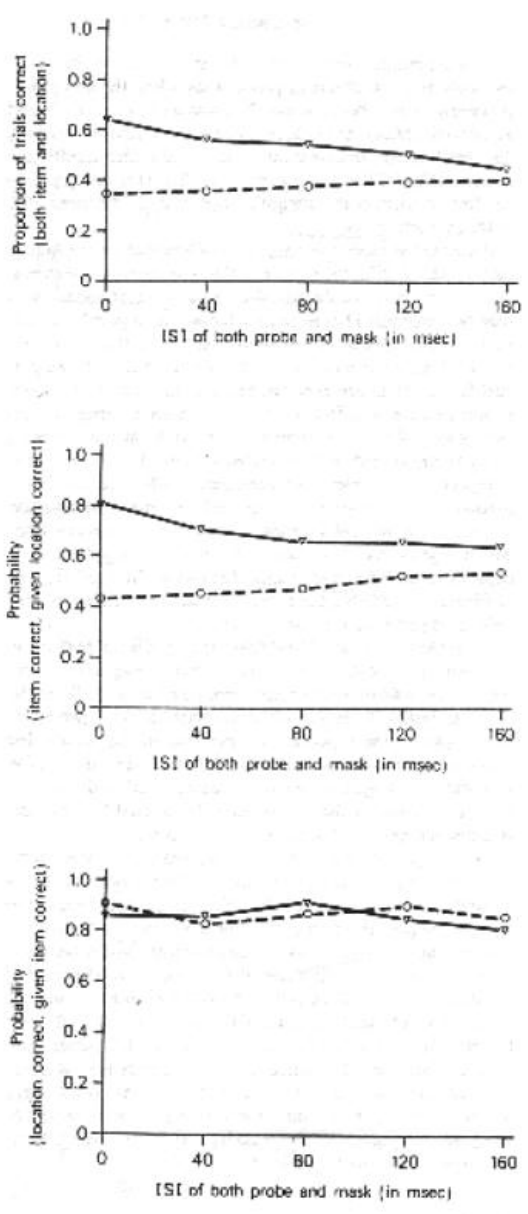

" No masking

o-----o Mask present

Figure 3. Currect recall of hoth inem ideutity abd its intra arruy hecation

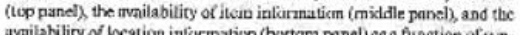

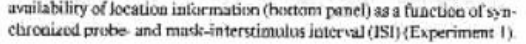

\section{Discussion}

My contention is that intralist and extralist intrusion errors are ambiguous indices of loss of location information and loss of item information, respectively. For this reason the two types of errors were not used in deriving the experimental predictions of this study. Nonetheless, it is necessary to ensure that the experiments in this study are comparable to Mewhort et al.'s (1981) experiments in terms of these errors. For this reason, intralist and extralist intrusion errors have been reported for comparison purposes. As has been shown, when scored in terms of intralist and extralist intrusions, results from the no-masking and the masking conditions of this experiment are comparable to those obtained under Mewhort et al.'s (1981) Conditions 1 and 2, respectively.

The proportion of trials in which both item identity and its location were correctly recalled was treated as a measure of partial-report performance. In terms of the new index 
of the availability of location information, the systematic decline of partial report in the absence of masking was not matched by a systematic decrease in location information as ISI increased. On the contrary, the availability of location information was not related to the delay of either the probe or the mask. Instead, the systematic decline in correct performance was matched by a systematic decrease in the availability of item information in the absence of masking.

The theoretical expectations of the orthodox view of iconic memory, as depicted in the two right-hand panels of Figure 1 are met by the data shown in the middle and bottom panels of Figure 3. That is, whereas location information does not change, item information declines systematically with increases in ISI. This is consistent with the select-then-identify mode of operation implied in Coltheart's (1980) and Treisman and Gelade's (1980) models.

\section{Experiment 2}

This experiment was designed to test the dual-buffer model by examining the effects of probe delay when the amount of processing time is held constant by using a fixed stimulus onset asynchrony (SOA). As has been noted earlier although masking disrupts location information in the dual-buffer model, the manifestation of this effect may be qualitatively different, depending on whether the disruption occurs in the feature buffer or the character buffer.

If the (SOA). is short, the disruption of location information is said to take place in the feature buffer. The stimulus identification process is hence disrupted because some of the features may be displaced. That is, the availability of item information suffers. This expectation has been depicted in the middle left panel of Figure 4 in terms of $p(\mathrm{I} \mid \mathrm{L})$. More specifically, two parallel flat functions are expected because the delay of the probe is said to have no effect on item information. Given a sufficiently long (SOA). only location information at the character buffer is interrupted. When coupled with the delay of the partial-report probe (whose effect is assumed to affect location information only), the mask is expected to lower the absolute amount of location information. That is, two parallel functions with a negative trend are expected. This expectation has been depicted in the bottom left panel of Figure 4. The top left panel of Figure 4 represents the expected systematic decline of partial-report performance with increases in ISI.

From Mewhort et al.'s (1981) description, it is not possible to determine if an (SOA). of $100 \mathrm{~ms}$ is sufficiently long to complete the transfer of feature information from the feature buffer to the character buffer. If this (SOA). is not long enough for the process to be completed, the expectations described in the middle left panel of Figure 4 should follow. On the other hand, if this (SOA). is of sufficient length, the expectations depicted in the bottom left panel of Figure 4 should be obtained. The dual-buffer model is in doubt if neither of these expectations is met. 
Only the partial-report probe was delayed in this experiment. When masking was applicable, the masking array was always presented at the immediate offset of the array, regardless of the delay of the partial-report probe. For this reason, a duration of $100 \mathrm{~ms}$ was used so as to avoid the possibility of a floor effect. Moreover, the interval between the probe delay and the offset of the stimulus array will be called probe-ISI when Experiment 2 is discussed. The specific values of the five probe-ISI levels were 0, 150, 250,500 , and $750 \mathrm{~ms}$. Longer probe-ISI values were used here because information in the iconic store has variously been estimated to last between 250 and $500 \mathrm{~ms}$. When there was no masking, the condition was analogous to Mewhort et al.'s (1981) Condition 1. The masking condition in this experiment was not run by Mewhort et al. (1981).

\section{Results}

The subjects' intralist and extralist intrusions under the various treatment combinations are depicted in the left and right middle panels of Figure 2 for comparison purposes (to Mewhort et al.'s, 1981, study) even though they do not play any role in the theoretical expectations of this experiment. In the case of intralist intrusions, both the effects of masking, $F(1,13)=5.42$, and of probe-ISI, $\mathrm{F}(4,52)=5.01$, were significant; so was the interaction between masking and probe-ISI, $F(4,52)=5.85$. In terms of extralist intrusions, only the main effects of masking, $\mathrm{F}(1,13)=72.45$, and of probe-ISI, $\mathrm{F}(4,52)$ $=5.92$, were significant. There was no interaction.

The subjects' partial-report performance as measured in terms of correct recall of both item identity and its location has been depicted in the top right panel of Figure 4 . As may be seen from the figure, masking reduced performance. Moreover, the subjects' performance declines as probe-ISI increases in the presence of the mask. The ANOVA revealed that the main effect of masking was significant, $F(1,13)=53.38$; so was the main effect of probe-ISI, $F(4,52)=11.79$. The Masking $x$ Probe-ISI interaction was also significant, $\mathrm{F}(4,52)=5.21$.

Masking reduced the availability of item information (see middle panel of Figure 4 ), but enhanced the availability of location information (bottom panel of Figure 4). The availability of item information decreased with increases in the probe-ISI of the partial-report probe. The ANOVA showed that both the main effects of masking and of probe-ISI were significant; for masking, Fail, 13) $=193.97$; for probe-ISI, F $(4,52)=$ 10.31. The analysis for the availability of location information revealed only a significant masking effect, $\mathrm{F}(1,13)=12.98$.

\section{Discussion}

As has been noted, the masking condition used in this experiment was not found in Mewhort et al.'s (1981) study. In the case of the no-masking condition, the subjects' extralist and intralist intrusion errors bore a functional relation with probe-ISI similar to 
that found in Mewhort et al.'s (1981) Condition 1, as may be seen from the middle two panels of Figure 2.

The fact that the masking array was always present at the immediate offset of the array, when applicable, ensured that the (SOA). was a constant one, namely, $100 \mathrm{~ms}$. In Mewhort et al.'s (1981) account, for short SOAS backward masking might disrupt the location arrangement of the features in the feature buffer, thereby producing item errors. Hence, masking should reduce the availability of item information because fewer correct characters are transferred into the character buffer. That is, although the absolute amount of information in the feature buffer is not affected by the mask, the absolute level of information is reduced by masking if the character buffer is utilized.

That is, in terms of the availability of item information (the middle left panel of Figure 4), a flat function across probe-ISI levels is prescribed by the dual-buffer model for the masking condition in this experiment. This should be the case because (a) the probe-ISI manipulation here was applicable to the partial-report probe only, and (b) the delay of the partial-report probe disrupts only the usefulness of the location information in the dual-buffer model. The function for the no-masking condition is expected to be parallel to the flat function of the masking condition, but with a higher intercept. The expectation of having two flat functions was not met. Instead, the availability of item information decreased with increases in the delay of the partial-report probe in the absence of masking.

Two alternatives have to be considered. First, the dual-buffer model is not supported by the results of this experiment. Alternatively, the 100-ms SOA might be more than sufficient to complete the transfer process (of features from the feature buffer into the character buffer). However, the second alternative can be rejected in view of the analysis in terms of $p(\mathrm{~L} \mid \mathrm{I})$.

Mewhort et al. (1981) also suggested that backward masking might disrupt the location information of the characters in the character buffer. Consequently, in terms of the availability of location information, the probe-ISI function under the masking condition should be parallel to the probe-ISI function without backward masking, but with a smaller absolute value. Moreover, both functions should have a negative trend. However, the two probe-ISI functions in the bottom right panel of Figure 4 are more or less flat. The absence of any probe-ISI effect on the availability of location information is contrary to the expectation of Mewhort et al.'s (1981) model that location information is lost or transposed when the partial-report probe is delayed.

The fact that the presence of the masking array (which stayed on until the subjects had responded) enhanced the recall of the location information is not surprising. Recall that the masking array was always at the immediate offset of the stimulus array when applicable. The masking array effectively extended the availability of the intra-array location information. 

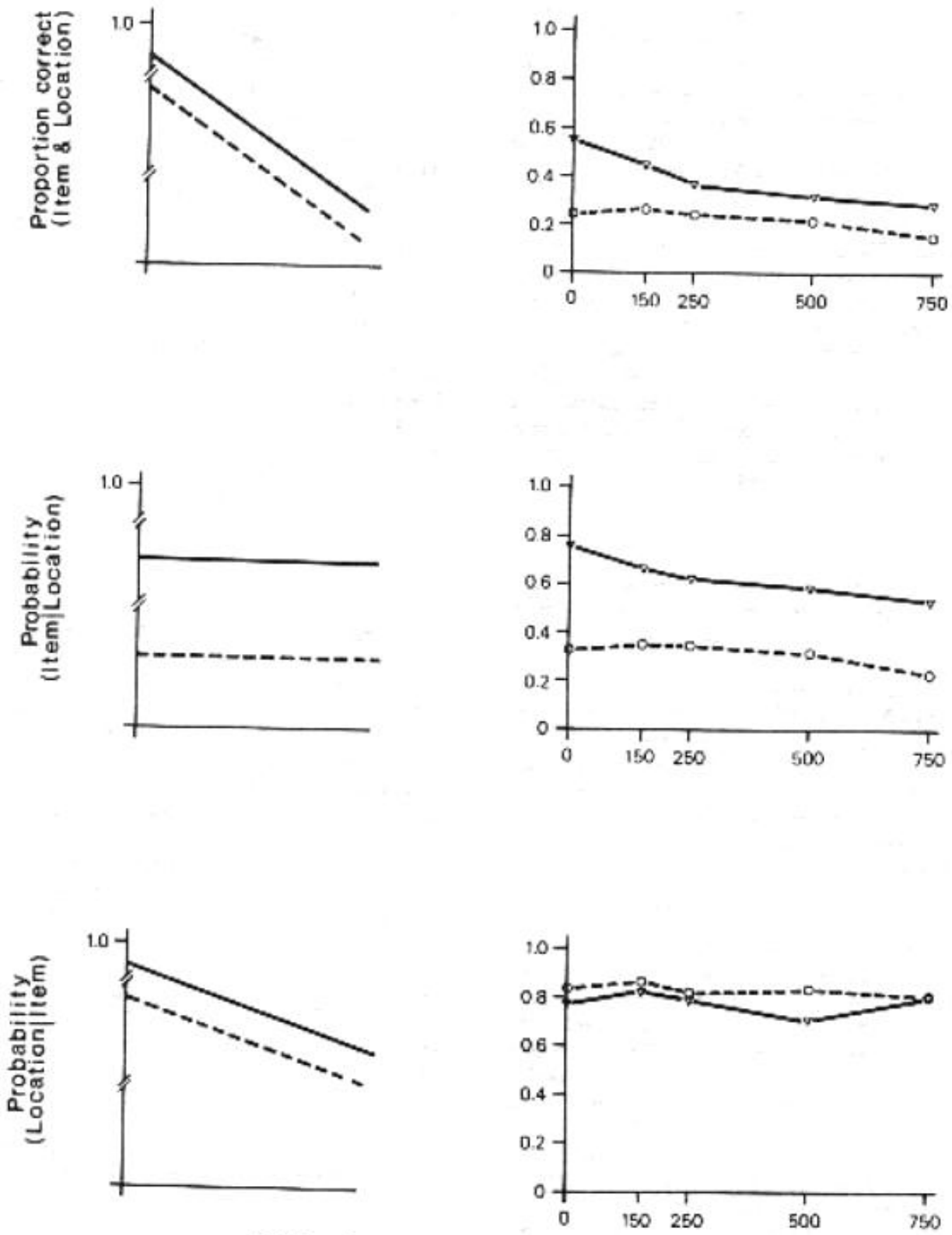

\section{ISI OF PROBE (MSEC)}

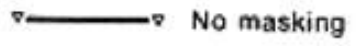

O-no Mask presented at ISI $=0$ msec. duration $=100 \mathrm{msec}$

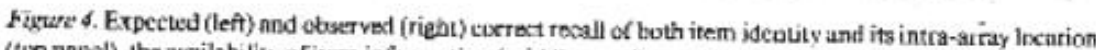
(twp pased), the availability of item informstion (middle penet), and the avsilability of Jocation information (botion panei) as a function of prube-IST wbin the mask was presented at the immediate offset of the stimulus in texperiment 2 


\section{Experiment 3}

This experiment was designed to consider the dual-buffer model by examining the effect of masking when uncertainty regarding which letter to recall was kept at a constant minimum. From what is known about backward masking, partial-report performance is expected to increase with increases in mask-ISI (Kahneman, 1968; Turvey, 1973).

As envisaged in the dual-buffer model, the progressive improvement with increases in the mask-ISI is due to the fact that more and more items are being successfully read from the feature buffer into the character buffer. Hence, the expected outcomes in terms of item information have been depicted in the middle left panel of Figure 5. Essentially, at short mask-ISI less item information should be available with than without a mask. However, it should reach a maximum asymptote at longer mask-ISI.
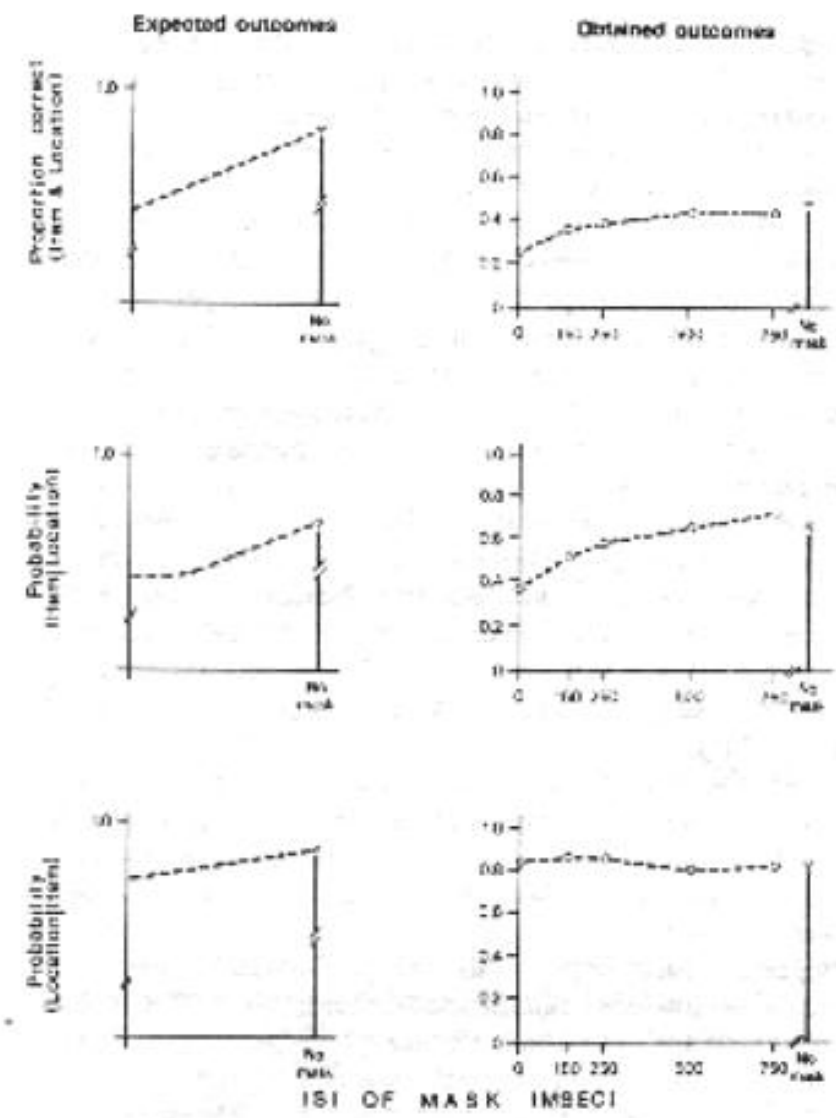

-...- Matk cressis.

Figare 5. Expected (left) and obecrwa (cight) recall of both jtoun iden. tiry aod its intra-array locatioe (top punel), the avnilability of item information (middle panci), and the availability of Jocation informatioe flottom panet) sa a function of mask-intorstimulus intersat (ISI) with the probe persented at the immediate alfset of the stamulus in ExperjIseat 3 , 
Some loss of location information at the level of the character buffer as a result of masking is, nonetheless, envisaged in the dual-buffer model. Consequently, the mask-ISI function in terms of $p(\mathrm{~L} \mid \mathrm{I})$ is expected to rise with increases in mask-ISI, as may be seen from the bottom left panel of Figure 5. The top left panel of Figure 5 reflects the expected partial-report performance as a function of mask-delay in terms of the dual-buffer model.

The partial-report probe was always presented at the immediate offset of the array in Experiment 3. The mask-ISI factor was applicable to the masking manipulation on half the occasions. For this reason, the ISI will be called mask-ISI when Experiment 3 is discussed. The five mask-ISI levels were $0,150,250,500$, and $750 \mathrm{~ms}$. The no-masking condition was analogous to Mewhort et al.'s (1981) Condition I when mask-ISI $=0 \mathrm{~ms}$, whereas the masking condition was analogous to their Condition 4 . The stimulus duration was $20 \mathrm{~ms}$.

\section{Results}

Backward masking was not present in half of the session. For the purpose of data analysis, the no-masking condition was treated as the control level of the mask-ISI manipulation. That is, data were analyzed with a one-way ANOVA in which there were six levels of mask-ISI.

In the two bottom panels of Figure 2 are depicted the subjects' intralist and extralist intrusion errors. They should be compared to Mewhort et al.'s (1981) findings under their Condition 4. By and large, the present observations are not inconsistent with their findings in terms of intralist and extralist intrusion errors. In the case of both extralist and intralist intrusions, the mask-ISI effect was significant; for intralist intrusions, $F(5,65)=6.05$; for extralist intrusions, $F(5,65)=4.98$.

The subjects' partial-report performance in terms of correct recall of both item identity and its intra-array location has been graphically depicted in the right top panel of Figure 5. Performance increased with increases in mask-ISI under the masking condition. The statistical analysis showed a significant mask-ISI effect, $\mathrm{F}(5,65)=12.27$.

The availability of item information increased with increases in mask-ISI in the presence of a mask (see the middle right panel of Figure 5). This is confirmed by a significant mask-ISI effect, $F(5,65)=19.26$. The mask-ISI manipulation did not have any effect on the availability of location information, as may be seen from the bottom right panel of Figure 5.

\section{Discussion}

As in Experiments 1 and 2, the functional relation between (a) the subjects' intralist intrusion errors and mask delay and (b) the subjects' extralist intrusion errors and mask delay were very similar to those found by Mewhort et al. (1981). 
The fact that the partial-report probe was presented at the immediate offset of the array ensured that there was minimal uncertainty regarding which item to recall. It follows from the dual-buffer model that the availability of location information should increase with increases in the mask-ISI of the masking array. Yet, there was not any mask-ISI effect on the availability of location information in the presence of masking.

The absence of an asymptote in terms of the availability of item information as mask-ISI increased is contrary to Mewhort et al.'s (1981) contention that backward masking interferes with location information at the level of the feature buffer at short SOA range. This finding supports the view that backward masking interrupts item identification (Turvey, 1973).

\section{General Discussion}

It has been suggested that the partial-report superiority over whole report is the result of an artifact brought about by the fact that ". . . measure is heavily biased against full report ..." (Mewhort \& Butler, 1983, p. 32). This bias is presumably due to the fact that the nature of response organization is different in whole and partial report (see also Dick, 1971). Mewhort et al. (1981) also argue that the decline in partial-report superiority with increases in mask-ISI reflects a progressive loss of useful location information. This latter claim relies on the demonstration that the systematic decline in partial report is complemented by a progressive increase in location errors.

The conditional probabilities, $p(\mathrm{I} \mid \mathrm{L})$ and $p(\mathrm{~L} \mid \mathrm{I})$, were used as the measures of the availability of item information and of location information, respectively. These conditional probabilities were preferred to $I$ (i.e., the correct recall of item identity) and $L$ (i.e., the correct recall of the item's intra-array location) because the latter two are ambiguous at the theoretical level. Moreover, specific expectations can be derived from both the dual-buffer model and the traditional view of iconic memory in terms of these two conditional probabilities. One likely problem with these measures is that the subjects might have guessed correctly either the item identity or its location on an unknown proportion of trials. How would guessing affect these measures?

The numerator of both of these conditional probabilities is the probability of recalling correctly both the item's identity and its location. The likelihood of getting both the identity and location of the item correct by guessing may reasonably be assumed to be negligible when compared to guessing either the identity of the item or its location. Hence, it is reasonable to assume that guessing may have only a negligible effect on the numerator of either of the two conditional probabilities. To the extent that either $p(\mathrm{~L})$ or $p(\mathrm{I})$ was inflated by guessing, $p(\mathrm{I} \mid \mathrm{L})$ or $\mathrm{p}(\mathrm{L} \mid \mathrm{I})$ would be reduced. This, however, would not affect the qualitative outcomes (i.e., the relation between the ISI functions with and without masking) of this study if guessing was independent of the ISI manipulation. The situation is more complicated if guessing was dependent on the ISI manipulation. 
Assume that guessing became more important as the task became more difficult. First, consider Experiment 3, in which the probe was always presented at the immediate offset of the stimulus array. It seems reasonable to assume that guessing would not affect $p(\mathrm{~L})$ because there was virtually no location uncertainty. The difficulty of the task was determined solely by how closely the mask followed the stimulus array. The "genuine" probability of recalling item identity may be called $p^{\prime}(\mathrm{I})$ in order to distinguish it from $p(\mathrm{I})$ (the observed probability of recall of item identity). The reasonable assumption here is that $p^{\prime}(\mathrm{I})$ was smaller than $p(\mathrm{I})$ at short mask-ISIs. That is, the "genuine" $p(\mathrm{~L} \mid \mathrm{I})$ at short mask-ISIs might have been underestimated as a result of guessing. Consequently, the left-hand end of the dotted-line function in the right bottom panel of Figure 5 should have been higher than it currently is. However, such an elevation of the dotted-line function might not be sufficient to turn the function into one with a significant negative slope. In any event, the data would still be inconsistent with the expectation of the dual-buffer model.

The mask (when applicable) was presented at the immediate offset of the stimulus array in Experiment 2. Only the delay of the probe was varied. It is reasonable to assume that guessing became more important at longer probe-ISIs. That is, $p^{\prime}(1)$ is smaller than $p(\mathrm{I})$, and $p^{\prime}(\mathrm{L})$ is smaller than $p(\mathrm{~L})$ at longer probe-ISIs. Consequently, the right-hand ends of all the functions in the middle and bottom panels on the right of Figure 4 might have to be elevated. However, the two functions in the right bottom panel of Figure 4 would still be inconsistent with the expectation of the dual-buffer model.

The situation is more complicated in the case of Experiment 1, in which the delay of the probe and of the mask were manipulated simultaneously. Delaying the mask should reduce the contribution of guessing because the task became easier at longer mask-delays. At the same time, delaying the probe would render the task more difficult. The dual-buffer model is not explicit regarding the relative importance of mask delay and probe delay. The reasonable assumption seems to be that the two may cancel each other out. Given this assumption, the data as depicted in Figure 3 would remain unchanged.

Some probable effects that guessing might have on the outcomes of the three experiments have been considered. In all cases, it seems reasonable to suggest that guessing could not have biased the outcomes in ways which are unfavorable to the dual-buffer model.

In terms of the new measures, it was demonstrated that the systematic decline in partial report was matched by a systematic decrease in item information when the partial-report probe was delayed. When performance improved with increases in the delay of the masking array, the improvement was matched by an increase in the availability of item information. Apart from not vindicating the dual-buffer model, this study also serves to exclude Mewhort et al.'s (1981) attempt to question the empirical foundation of iconic memory in the sense of informational persistence. 
Crucial to the valid application of the partial-report task is the assumption that the select-then-identify strategy (called the "selective readout" by Coltheart, 1980) is adopted by the subjects. (See also Treisman \& Gelade, 1980, for a similar position.) However, some investigators have argued that the selection takes place after the identities of the briefly presented items have been established (Dick, 1969, 1971; Merikle, 1980; Mewhort et al., 1981; Van der Heijden, 1984).

Dick $(1969,1971)$ and Merikle (1980) obtained data consistent with the identify-then-select strategy and with Sperling's (1960) partial-report task. However, their subjects were not given any meaningful training on the partial-report task. Subjects could use the select-then-identify mode of processing only when they were given at least an hour's training on the partialreport task (Chow, 1985). The observations made in this series of experiments support the select-then-identify assumption.

Mewhort et al.'s (1981) dual-buffer model has recently been examined by Van der Heijden (1984), who is also critical of the orthodox view of iconic memory as represented by Averbach and Coriell (1961) and Coltheart (1977). Van der Heijden's (1984) version of a dual-buffer model consists of Store A (in which only precategorical information is available) and Store B (in which postcategorical information is stored). The distinguishing marks of Van der Heijden's (1984) and Mewhort et al.'s (1981) models are as follows:

1. Although only visual features (such as straight lines, angles, and the like) are allowed in Mewhort et al.'s (1981) feature model, logogens of letters and of words, in addition to visual features, are also allowed in the precategorical Store A in Van der Heijden's (1984) model.

2. Selection under the partial-report task constraint is achieved by a process called precategorical filtering at the level of Store A. In other words, Van der Heijden (1984) argued for the identify-then-select option. However, this selection is done before Store B (a store analogous to Mewhort et al.'s, 1981, character buffer).

How can the difference between Van der Heijden's (1984) and the present conclusions be reconciled? The identify-then-select versus the select-then-identify issue cannot be discussed meaningfully without first identifying the level of abstraction. For example, transducing electromagnetic energy into a color sensation, extracting an angle from the letter A, and identifying the letter A as A are all information processing activities. However, they differ in sophistication. Traditionally, in the context of the information-processing approach to visual perception (e.g., Haber \& Hershenson, 1980), transducing a particular frequency of electromagnetic energy into a color sensation is not treated as an act of categorization. As a methodological assumption, many investigators treat color as a precategorical dimension when they study iconic memory (e.g., Clark, 1969; Turvey \& Kravetz, 1970; von Wright, 1968, 1970). That is, color information is 
assumed to be available in the iconic store. Van der Heijden's (1984) argument can now be considered.

Van der Heijden (1984) rejected the orthodox view of iconic memory because his subjects' partial-report performance was affected by the irrelevant stimulus items in the partial-report display. However, the identities of both the relevant and the irrelevant stimulus items in his study were defined solely in terms of color, a dimension that is generally accepted to be a precategorical dimension available at the iconic level. Moreover, naming a color does not involve building a character from some discrete features. For this reason, it can be suggested that Van der Heijden's (1984) choice of stimulus has precluded him from studying iconic memory in the sense of Coltheart's (1980) informational persistence.

In demonstrating that information at the iconic level is nonassociative, Wickelgren and Whitman (1970) effectively showed that information in the probed location was independent of the information in any other location if the choice of stimulus material permitted the study of informational persistence. In other words, Van der Heijden's (1984) data are not inconsistent with the orthodox view that selection can be made at the iconic level if the criterion of selection is color.

Unlike Mewhort et al. (1981), Van der Heijden (I 984) found that as partial-report performance declined with increases in mask-ISI, both item information and location information also declined systematically with increases in mask-ISI. There are two reasons why Van der Heijden's (1984) data are not necessary inconsistent with either Mewhort et al.'s (1981) results or those of this series of experiments. Relevant to Mewhort et al.'s (1981) findings is the previous comment that Van der Heijden's (1984) choice of stimulus material necessarily precluded his subjects from engaging in forming characters from features, a process necessary for Mewhort et al.'s (1981) task. This comment is also applicable to the present study. Pertinent only to this series of experiments is the fact that Van der Heijden (1984) essentially treated intralist and extralist intrusion errors as indices of item and of location information, respectively. This is the methodological assumption being questioned here.

Certain derivations have been made from Mewhort et al.'s (1981) dual-buffer model even though the formulation of the model is not as explicit as expected. The derivations were based primarily on the identify-then-select assumption. The dual-buffer model was assessed in terms of two conditional probabilities when (a) the delay of the partial-report probe was synchronized with the delay of the mask (Experiment 1), (b) only the delay of the probe was varied when the mask was presented at the immediate offset of the stimulus (Experiment 2), and (c) only the delay of the mask was varied when the probe was presented at the immediate offset of the stimulus (Experiment 3). In all cases, the theoretical expectations of the dual-buffer model were not met. Instead, the results were consistent with the traditional view of iconic memory, particularly the selectthen-identify implication. 


\section{References}

Averbach, E., \& Coriell, A. S. (1961, January). Short-term memory in vision. Bell System Technical Journal, 40, 309-328.

Campbell, A. J., \& Mewhort, D. J. K. (1980). On familiarity effects in visual information processing. Canadian Journal of Psychology, 34, 134-154.

Chow, S. L. (1985). Iconic store and partial report. Memory \& Cognition, 13, 256-264.

Chow, S. L. (in press). Iconic memory or icon? Behavioral and Brain Sciences.

Clark, S. E. (1969). Retrieval of colour information from preperceptual memory. Journal of Experimental Psychology, 82, 263-266.

Coltheart, M. (1975). Iconic memory: A reply to Professor Holding. Memory \& Cognition, 3, 42-48.

Coltheart, M. (1977). Contemporary models of the cognitive processes: I. Information about input and storage. In V Hamilton \& M. D. Vernon (Eds.), The development of cognitive processes (pp. 11-41). London: Academic Press.

Coltheart, M. (1980). Iconic memory and visible persistence. Perception \& Psychophysics, 27, 183-288.

Dick, A. O. (1969). Relations between the sensory register and shortterm storage in tachistoscopic recognition. Journal of Experimental Psychology, 82, 279-284.

Dick, A. O. (1971). On the problem of selection in short-term visual (iconic) memory. Canadian Journal of Psychology 25, 250-263.

Haber, R. N. (Ed.). (1969). Information processing approaches to visual perception. New York: Holt, Rinehart \& Winston.

Haber, R. N. (1971). Where are the visions in visual perception? In S. J. Segal (Ed.), Imagery: Current cognitive approaches (pp. 33-48). New York: Academic Press.

Haber, R. N. (1983). The impending demise of the icon: A critique of the concept of iconic storage in visual information processing. Behavioral and Brain Sciences, 6 , $1-11$.

Haber, R. N., \& Hershenson, M. (1980). The psychology of visual perception (2nd ad.). New York: Holt, Rinehart \& Winston. 
Haber, R. N., \& Nathanson, L. S. (1968). Post-retinal storage? Some further observations on Parkes' camel as seen through the eye of a needle. Perception \& Psychophysics, 3, 349-355.

Haber, R. N., \& Standing, L. G. (1969). Direct measures of short-term visual storage. Quarterly Journal of Experimental Psychology, 21, 4354.

Holding, D. (1970). Guessing behaviour and the Sperling store. Quarterly Journal of Experimental Psychology, 22, 248-256.

Holding, D. (1972). Brief visual memory of English and Arabic letters. Psychonomic Science, 28, 241-242.

Holding, D. (1975). Sensory storage reconsidered. Memory \& Cognition, 3, 31-41.

Kahneman, D. (1968). Method, findings, and theory in studies of visual masking. Psychological Bulletin, 70, 404-425.

Loftus, G. R. (1983). The continuing persistence of the icon. Behavioral and Brain Sciences, 6, 28.

Merikle, P. M. (1980). Selection from visual persistence by perceptual groups and category membership. Journal of Experimental Psychology: General, 109, 279-295.

Mewhort, D. J. K., \& Butler, B. E. (1983). On the nature of brief visual storage: There never was an icon. Behavioral and Brain Sciences, 6, 31-33.

Mewhort, D. J. K., \& Campbell, A. J. (1978). Processing spatial information and the selective-masking effect. Perception \& Psychophysics, 24, 93-100.

Mewhort, D. J. K., Campbell, A. J., Marchetti, F M., \& Campbell, J. I. D. (1981). Identification, localization, and "iconic memory": An evaluation of the bar-probe task. Memory \& Cognition, 9, 50-67.

Neisser, U. (1967). Cognitive psychology. New York: Appleton-CenturyCrofts.

Neisser, U. (1976). Cognition and reality: Principles and implications or cognitive psychology. San Francisco: W. H. Freeman.

Sakitt, B. (1975). Locus of short-term visual storage. Science, 190, 1318-1319.

Sakitt. B. (1976a). Iconic memory. Psychological Review, 83, 257-276. 
Sakitt, B. (1976b). Psychological correlates of photoreceptor activity. Vision Research, $16,129-140$.

Sperling, G. (1960). The information available in brief visual presentations. PsychologicalMonographs, 74(11), 1-29.

Treisman, A. M., \& Gelade, G. (1980). A feature-integration theory of attention. Cognitive Psychology, 12, 97-136.

Turvey M. T (1973). On the peripheral and central processes in vision: Inferences from an information-processing analysis of masking with patterned stimuli. Psychological Review, 80, 1-52.

Turvey, M. T, \& Kravetz, S. (1970). Retrieval from iconic memory with shape as the selection criterion. Perception \& Psychophysics, 8, 171172.

Van der Heijden, A. H. C. (1984). Postcategorical filtering in a bar-probe task. Memory \& Cognition, 12, 446-457.

von Wright, J. M. (1968). Selection in visual immediate memory. Quarterly Journal of Experimental Psychology, 20, 62-68.

von Wright, J. M. (1970). On selection in visual immediate memory. Acta Psychologica, 33, 280-292.

Wickelgren, W. A., \& Whitman, P. T (1970). Visual very-short-term memory is nonassociative. Journal of Experimental Psychology, 84, 277-281.

This research was supported by a Category A grant from the University of Wollongong to the author. I wish to thank Don Mixon for his advice. I am also grateful to William Banks, Geoffrey R. Loftus, and two anonymous reviewers for their helpful comments.

Correspondence concerning this article should be addressed to Sin L. Chow, Department of Psychology, University of Wollongong, PO. Box 1144, Wollongong, New South Wales, Australia, 2500. Now at the Department of Psycholgy, University of Regina, Regina, Saskatchewan, Canada S4S 0A2 